equivalents for such groups as the hawks and doves within its limits. Whereas it seems to me that the truer parallel is between the whole class Insecta and Birds, and that the equivalent groups for hawks, doves, \&c., are to be looked for, not in one of the sections, but in the whole of the class. He looks for both hawks and doves in the Lepidoptera. I find nothing but doves. If you want hawks you must go to the dragon-flies, which are their equivalent; and, of course, if we are only dealing with doves, there is nothing in the known phenomena of hybridisation opposed to such a cross having taken place.

It is impossible in the brief space that you would allow me, even to glance at the many arguments that I could adduce to show that this is the true position of the Lepiloptera. I hope to do so elsewhere. But I would only remind entomologists, especially lepidopterists, of the trifling characters on which their renera have been established, and how difficult it has been to find any generic characters at all. This is frankly acknowledged as the great difficulty attending the study of Lepidoptera, consequently characters which wquld never for a moment be looked on as generic in any other group of animals, are there allowed that value. If any specialist in another group objects, what is the answer? "We have no better characters, and we must do the best we can with the slight ones we possess." Quite right, in a systematic point of view. If the species of doves came to be reckoned by thousands, the ornithologist would just have to do the same thing; but that would not alter the position of doves in the animal kingdom-they would still bear the same relation that they do now to hawks, and be equally open to hybridisation among themselves, indeed, more so; for such great numbers of one type would be a presumption in favour of every mode by which species could be increased having been resorted to ; and this by the way is an additional indirect argument in favour of hybridisation sometimes taking place among Lepidoptera.

Of course, I do not mean to say that there is nothing more than specific distinction between the Danaids and Nymphalids. I recognise them as good genera, but only as genera sufficiently nearly akin to allow of hybridisation taking place between themand ecce signum-the mimics in question partaking of the characters of each in all respects as other hybrids do.

ANDREW MURRAY

67, Bedford Gardens, Kensington, Dec. 30, I 870

\section{Measurement of Mass}

THE favourite definition of mass in the text-books seems to be that the mass of a body is the quantity of matter it contains. If we had to do with but one kind of matter this would be intelligible, but I am at a loss to know what is meant when it is said that a piece of cork contains as much matter as a piece of lead. The only satisfactory method of explaining what is meant by the mass of a body, is to define it as a constant belonging to the body, which expresses the proportion between the force (measured statically) acting upon it and the acceleration produced ; that every body has such a constant is the result of experiment. The mass of a body has no necessary connection with its weight. We employ weight to measure mass simply because gravity is a convenient constant force. If then we adopt a pound as our unit of weight, and use $g$ to denote the force of gravity in reference to a foot and a second as the units of length and time, our unit of mass becomes the mass of $g$ pounds, and this is not variable, although the unit of weight employed is variable; since if a true pound, as determined at London, were carried to the North Pole, it would weigh more than a pound, precisely in the proportion in which gravity at the Pole is greater than gravity at London.

The Reviewer of EveretT's "Deschanel"

\section{PHOTOGRAPHIC PROCESSES OF THE PRESENT DAY}

THE last two or three years will certainly mark an era in Photography, for not only have several novel and important printing methods been discovered during that period, but other processes of less recent origin have of late been so elaborated and improved as to have become at the present moment practical and easy of manipulation. All of these are, without exception, based upon the action of light upon the bichromates of potash and ammonia; in no single case is the use of a silver salt involved-the agent employed for securing the photographic image in ordinary paper printing-and this is, in truth, a point whose value cannot be too greatly insisted on ; for the silver print, be it washed and freed as thoroughly as possible from any deleterious bodies, will always suffer, more or less, from attacks of an impure atmosphere, the delicate metallic film of which the image consists being peculiarly liable to change, from the sulphur compounds and other impurities not unfrequently contained in the air we breathe. And even those silver pictures which do not at first show actual traces of fading or discoloration, will very soon be found, on careful examination, to have parted with some of their original brilliancy, and to lack the pristine freshness which always characterises newlyproduced albumenised prints.

It is a great step onwards, then, to have at our disposal practical processes in which the employment of silver may be altogether dispensed with, by the substitution of another material of a more permanent character, either in the form of a chromium compound, or, what is better still, in the shape of gelatinous or greasy ink; and so clear and promising does the photographic horizon appear just now in this direction, as to leave little ground for doubting that before long the practice of printing in silver will be generally abandoned.

All recent printing processes rest, as we have before said, on the action of light upon the bichromates, and here we would parenthetically refer to a simple and familiar experiment which will help very materially to simplify our subsequent remarks. The well-known plan pursued by school-boys for printing fern-leaves and other objects by the aid of the sun, will readily be called to mind by many of us, and this simple manipulation it is that forms the groundwork of the whole series of inventions before us. A sheet of ordinary paper, which has of course been sized, or, in other words, received a thin coating of gelatine, is rubbed over with a solution of bichromate of potash; the latter, as we know, when mixed with any organic body renders the same sensitive to light, and the sizing or gelatine upon the paper becomes in this way endowed with excitable properties. Having been dried in the dark, our sheet of paper is next placed in the sun with the fern-leaf, or other object to be copied, pressed down upon it, and the light acting upon all such portions of the sheet as are not covered up, browns the gelatine there and renders it insoluble; the sizing underneath the leaf, and screened therefore from the light, escapes this reaction and remains soluble, and this, on the printing being completed and the paper washed in water, is at once dissolved away, there remaining a white image of the leaf upon a brown ground composed of bichromated gelatine rendered insoluble by the sun's rays. This experiment may be regarded as the key to the whole question of photographic printing, and by bearing it in mind the reader will have no difficulty in at once comprehending the various inventions of the kind just now being made public.

The first method claiming our attention is the so-called carbon process. Photographic printing of this nature in one form or another has been carried on probably for upwards of fifteen years; but in its experimental stage the mediocre character of the results furnished by it were such as to deprive the system of any material support from photographers, and until, in fact, Mr. J. W. Swan, of Newcastle, made known his method, no easy or reliable modus operandi can be said to have existed. The plan followed by Mr. Swan was to prepare a warm solution of gelatine and bichromate of potash mixed with some finely divided pigments, such, for instance, as Indian Ink, and apply this mixture in the form of a coating to a sheet of paper, 